\title{
DISPERSION EFFECTS OF FIBER BENDING PERFORMED ON MICROSTRUCTURED FIBER
}

\author{
Michal LUCKI \\ Department of Telecommunications Engineering, Faculty of Electrical Engineering, Czech Technical University in Prague, \\ Technicka 2, 166 27, Prague, Czech Republic, e-mail: lucki@fel.cvut.cz
}

\begin{abstract}
This paper deals with dispersion effects resulting from fiber bending performed on microstructured fiber. It is theoretically possible to achieve significant negative chromatic dispersion by fiber bending. Bending of photonic crystal fiber, performed at specific conditions, can result in coupling modes and non-standard dispersion properties. The presented knowledge should be taken into account during the design of photonic crystal fibers immune to bending. For example, the potential tune of the operating wavelength by the reason of bending should be avoided. Minimum values of negative chromatic dispersion and of a minimumdispersion wavelength can be controlled by the value of bending radius. Among others, dispersion diagrams obtained for different bending and for different air-filling fraction are presented.
\end{abstract}

Keywords: chromatic dispersion, fiber bending, Photonic Crystal Fiber

\section{INTRODUCTION}

The generally accepted view on fiber bending assumes that it is responsible for bending losses in any type of optical fiber. However, there are other phenomena that can be observed in bent Photonic Crystal Fiber (PCF), such as significant negative chromatic dispersion, nonlinear transmission characteristics of microstructured or the potential tune of operating wavelength. Theoretically, multiple winding onto a reel can result in broadband negative dispersion, since each winding is done at different reel radius. Nevertheless, this paper does not have as its aim to suggest a new method for signal recovery by using anomalous dispersion. The goal is to present non-standard dispersion properties being the result of bending and describe the mechanism in more details. The presented knowledge could, for example, be taken into consideration in the design of an optical fiber, which is immune to bending. A complex study of bending results at telecommunication wavelengths is discussed. Fiber bending, performed on PCFs and considered in the context of negative dispersion at the C-band $(1530 \mathrm{~nm}-1565$ $\mathrm{nm}$ ), has not been studied, yet.

One of the problems addressed in this work is the study of mutual relations between minimum dispersion and the structural parameters of a solid-core PCF. By varying the hole pitch or the hole diameter in PCFs, it is possible to tune the value of minimum dispersion to the required wavelength. By now, it is confirmed for classical dual-core fibers, as presented by Fevrier et al [1].

The detailed content of problems addressed in this work contains modeling of light propagation in bent PCFs, as well as investigation of minimum dispersion at the $\mathrm{C}$ band, analysis of sensitivity of minimum dispersion and minimum dispersion wavelength to deviation in structural parameters.

The following chapter deals with works related to the problem of fiber bending, as well as to the current state of art, since the general problem of negative chromatic dispersion is concerned. Next chapter describes the method and the simulation procedure, used for obtaining results contained in this paper. Results and discussion are presented in chapter 4. The last part of this paper is focused on conclusions.

\section{RELATED WORKS}

Fiber bending is usually considered as an undesired phenomenon in the context of bending losses [2-5]. The topic is also related with the design of an optical fiber immune to bending [6]. There is a paper reporting the existence of oscillations in the dependence of bending losses upon bending radius, associated with coupling modes [7]. Last but not least, it is presented that bending can be used for coloring of white-light transmission [8]. Different colors appear, since different value of bending radius is responsible for propagation of different frequency components; it further means high wavelengthdependent losses. White-light transmission experiments showed that the fiber can be tuned to transmit specific colors of the visible spectrum by bending.

\subsection{Negative dispersion in Photonic Crystal Fibers}

One of the potential consequences of fiber bending is negative chromatic dispersion, which can be obtained only at the specific conditions, as far as structural parameters are concerned. Because of positive Group Velocity Dispersion (GVD), different frequency components of the transmitted signal are propagated at different velocities, which causes the pulse extension and limit the maximum distance of optical paths without signal recovery [9]. In the accordance to the ITU-T G.652 recommendation, the typical value of chromatic dispersion in Single Mode Fibers (SMF) can be $17 \mathrm{ps} \cdot \mathrm{nm}^{-1} \cdot \mathrm{km}^{-1}$ at the $1550-\mathrm{nm}$ wavelength [10]. Negative chromatic dispersion is used in telecommunications to compensate GVD, and consequently to allow for the increase of a bit rate.

Negative chromatic dispersion can be the result of the specific design of optical fiber geometry, for example in dual-core structures, eventually combined with doping of the fiber's core. In PCFs, concentric cores can be realized by having selected rings of holes absent [11]. The described PCF, created by seven dual cores (designed 
without doping), exhibits chromatic dispersion coefficient equal to $-600 \mathrm{ps} \cdot \mathrm{nm}^{-1} \cdot \mathrm{km}^{-1}$. (However, authors report that the largest negative chromatic dispersion that can be achieved in this design is $-55000 \mathrm{ps} \cdot \mathrm{nm}^{-1} \cdot \mathrm{km}^{-1}$ ). Last but not least, dual core technique, applied to dispersion compensation purposes in high-speed optical systems, is investigated in $[12,13]$. One of the highest ever-published values of negative chromatic dispersion is reported by Yang et al [14]. The compensating PCF with doped core is proposed by Zsigri et al in [15], dispersion is $-1350 \mathrm{ps} \cdot \mathrm{nm}^{-1} \cdot \mathrm{km}^{-1}$. However, doped PCFs with small core (the diameter is only $3 \mu \mathrm{m}$ ) suffer from small mode field area - the core must be small to provide strong confinement of the propagated light. Doping the PCF's core can also result in emerging of higher order modes [11]. Classical dual-core fibers can support the guidance of two super-modes, one characterized by negative chromatic dispersion and the other one by the positive chromatic dispersion, as concerned in [16]. In the considered structure, coupled light forms one cladding mode, resembling a sickle. On the contrary, coupled light in PCFs forms a gallery of cladding modes in the spaces between holes [17].

\subsection{Bending-induced negative chromatic dispersion}

There are publications reporting negative chromatic dispersion resulting from fiber bending performed on classical dual-core optical fibers [1]. The authors report negative dispersion in a classical dual-core fiber (i.e. stepindex with ring) associated with coupling modes; resultant value is $-1800 \mathrm{ps} \cdot \mathrm{nm}^{-1} \cdot \mathrm{km}^{-1}$. In dual-core fibers, this phenomenon is not used to compensate GVD, because of significant bending losses. On one hand, fiber designers should take this phenomenon into account during the design of new Dispersion Compensating Fiber (DCF). On the other hand, the mechanism could be employed for tuning the minimum-dispersion wavelength (or as well zero-dispersion wavelength) by macro-bending. This approach applies to PCFs, too [18]. Nevertheless, on the contrary to the classical dual-core fibers, PCFs can produce even one hundred times greater chromatic dispersion, as, for example, indicated in the following sections of this paper.

\section{METHODS}

The mechanism of fiber bending employs the theory of coupling modes, which creates the context for many scientific problems, such as the presence of super-modes in dual-core fibers for both: step-index with ring [16] and PCFs with one ring of holes absent [14].

\subsection{Theory of coupling modes}

Coupled mode theory is presented, for example, in $[19,20]$. In general, bent fiber can theoretically guide two coupling super-modes: the fundamental mode exhibiting negative chromatic dispersion and the cladding mode (practically, it is often a gallery of cladding modes) characterized by positive value of dispersion coefficient. The minimum dispersion is determined at the wavelength, for which the difference of the two values of effective index corresponding to the two super-modes is zero, which occurs at the phase-matching wavelength (1), as predicted by Auguste et al [21]:

$n_{\text {eff } 1}-n_{\text {eff } 2}=\left|n_{\text {eff } 1}-n_{\text {eff } 2}\right|=0$

Fiber bending could be represented by an equivalent refractive index profile, taking into account the value of bending radius $R$, as in formula (2):

$n_{2}(x, y)=n_{1}(x, y) \cdot\left(1+\frac{x}{R}\right)$

where $n_{l}$ is the refractive index of the material in $(x, y)$ position (unbent fiber), and $n_{2}$ is the refractive index of the material at the same point, modified by a factor taking into account the impact of bending (the adjustment of the $x$ position by the value of bending radius $R$, assuming the bending in the $x-z$ plane).

If the core is single-mode at $1550 \mathrm{~nm}$, with the electric field distribution $E_{l}(x, y)$, and the effective index is $n_{\text {eff }}$, the cladding mode (or summary intensity of cladding modes) emerging after bending is described by $E_{2}(x, y)$ and the effective index - by $n_{\text {eff } 2}$. Cladding modes in the spaces between holes are weakly confined and the resultant value of chromatic dispersion is smaller than the value for the core-mode. We consider two super-modes as the elementary modes of two independent guides. The electric field of the super-modes composed of the fundamental mode in the core and the modes guided in the cladding is specified, as in $(3,4)$ :

$$
\begin{aligned}
& \varepsilon_{I}=\psi_{I} \exp ^{-j \beta_{I} z} \\
& \varepsilon_{I I}=\psi_{I I} \exp ^{-j \beta_{I I} z}
\end{aligned}
$$

where $\psi_{i}$ is its radial distribution, $\beta_{i}$ is the propagation constant of the considered mode. To simplify the definition, we decompose the super-modes into elementary modes, as denoted in $(5,6)$ :

$$
\begin{aligned}
& \psi_{I}=b_{I 1} \psi_{1}+b_{I 2} \psi_{2} \\
& \psi_{I I}=b_{I I 1} \psi_{1}+b_{I I 2} \psi_{2}
\end{aligned}
$$

Modal coefficients are defined, as presented in (7-10):

$$
b_{I 1}=\frac{\frac{\beta_{1}}{2 \mu_{0} \omega} 2 \pi \int_{0}^{\infty} \psi_{I} \psi_{1} r d r}{P_{m 1}=1}=\frac{\int_{0}^{\infty} \psi_{I} \psi_{1} r d r}{\int_{0}^{\infty} \psi_{1}{ }^{2} r d r}
$$

$$
\begin{aligned}
b_{I 2}= & \frac{\int_{0}^{\infty} \psi_{I} \psi_{2} r d r}{\int_{0}^{\infty} \psi_{2}{ }^{2} r d r} \\
b_{I I 1}= & \frac{\int_{0}^{\infty} \psi_{I I} \psi_{1} r d r}{\int_{0}^{\infty} \psi_{1}{ }^{2} r d r}
\end{aligned}
$$




$$
b_{I I 2}=\frac{\int_{0}^{\infty} \psi_{I I} \psi_{2} r d r}{\int_{0}^{\infty} \psi_{2}{ }^{2} r d r}
$$

The amplitudes of the field of the first super-mode and the two elementary modes are positive in all points in the cross section. The second super-mode is positive, $b_{I 1}, b_{I 2}$, $b_{I I I}$ must be positive, and $b_{I I 2}$ negative. At the phasematching wavelength, the modal coefficients become, as described by the following equation:

$$
b_{I 1}=b_{I 2}=b_{I I 1}=-b_{I I 2}
$$

For wavelengths far from $\lambda_{0}$, we obtain equation (12) for $\Delta \beta<0$ or (13) for $\Delta \beta>0$ :

$$
\begin{aligned}
& b_{I 1}=1, b_{I 1}=0, b_{I I 1}=0, b_{I I 2}=-1 \\
& b_{I 1}=0, b_{I 2}=1, b_{I I 1}=1, b_{I I 2}=0
\end{aligned}
$$

The supermodes can be considered together close to the phase-matching wavelength $\lambda_{0}$ [16]. The field distribution can be resolved numerically, by using one of the simulation techniques, such as the Finite Difference Time Domain (FDTD) method [22] or the Finite Difference Frequency Domain (FDFD) method [23]. An overview of modeling techniques gives $\mathrm{K}$. Kawano et al [24].

\subsection{Simulation tool and procedure}

The simulation tool used for providing results contained in this work is a commercially available mode solver by Lumerical Solution Inc., implementing a fullvectorial FDFD method, which implements the algorithm similar to the one described in details by Zhu et al [25]. An index averaging technique is used for the cells across interfaces. The algorithm of index averaging implemented in the solver is based on a Yee's mesh algorithm of discretization [26]. The constant of propagation as a function of the wavelength can be evaluated by this algorithm. Having this, the effective index corresponding to the selected mode can be calculated as a quotient of constant of propagation and a free space wave vector. Group index, group velocity, and group delay can be obtained, too. Finally, GVD is calculated as the derivation of group delay, according to the relation (14):

$D=\frac{d \tau}{d \lambda} \cdot \frac{1}{L}\left[\mathrm{~s} \cdot \mathrm{nm}^{-1} \cdot \mathrm{km}^{-1}\right]$

where $L$ is the length of a fiber, and $\tau$ is the group delay of the propagated signal (15):

$\tau=\frac{1}{v_{G}}[\mathrm{~s}]$

Group velocity could be expressed by the following relation [27]:
$v_{G}=\frac{d \omega}{d k}\left[\mathrm{~m} \cdot \mathrm{s}^{-1}\right]$

\subsection{Investigated model of a microstructured fiber}

To create the model of PCF to study bending effects, a classical Index Guiding PCF consisting of a silica core in a regular array of holes, with the medium air-filling fraction and the small core diameter is concerned. Assumed refractive index of silica is 1.445 . A relatively big hole diameter and pitch is preferred (hole diameter $d$ approximately $11.7 \mu \mathrm{m}, d / \Lambda$ being roughly 0.504$)$. The core is single-mode at $1550 \mathrm{~nm}$, whereas the multi-mode solution in the cladding of a bent PCF is permitted. The cladding is multi-mode, because of the presence of holes that forbids for origination of one cladding mode.

The estimations of bending radius value for modeled structure indicates that bending radius of approximately $6.6 \mathrm{~cm}$ results in negative chromatic dispersion. Then, for assumed values of normalized hole diameter and the curvature radius, a wavelength-dispersion dependency is plotted. Greater curvature radius or greater normalized hole diameter could tune the minimum negative chromatic dispersion towards shorter wavelengths. For the values of bending radius far from this value, the structure exhibits a linear wavelength-dispersion relation. Next, it is possible to adjust hole diameter to receive negative chromatic dispersion at the C-band. The observation of minimum dispersion (and minimum-chromatic dispersion wavelength) as a function of bending radius or hole diameter is provided. The cross-section of the investigated PCF is presented in Fig. 1.

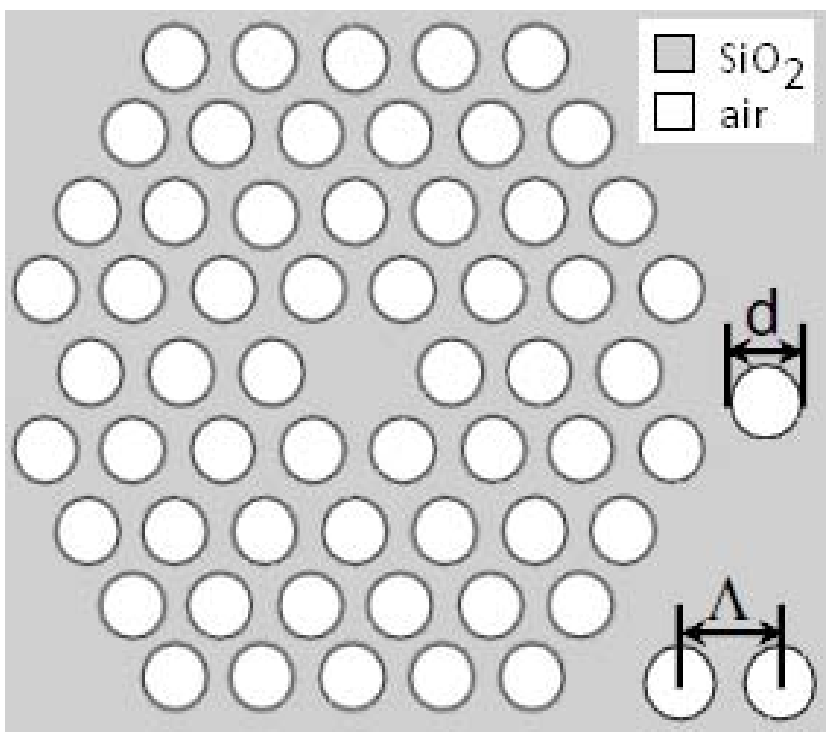

Fig. 1 Transverse structure of investigated bent microstructured fiber consisting of a silica core in a hexagonal cladding arrangement

The model values of the PCF's geometry used for further investigation, (observation of minimum dispersion and minimum dispersion wavelength), are presented in Table 1. 
Table 1 The model values of geometry of bent PCF for simulation of negative chromatic dispersion at the C-band

\begin{tabular}{|c|c|c|}
\hline & $\begin{array}{c}\text { Observation of } \\
\mathrm{d} / \Lambda \text { (variable) }\end{array}$ & $\begin{array}{c}\text { Observation of } \\
\mathrm{R} \text { (variable) }\end{array}$ \\
\hline $\begin{array}{c}\text { Minimum } \\
\text { dispersion }\end{array}$ & $\begin{array}{c}-48850 \\
\mathrm{ps} \cdot \mathrm{nm}^{-1} \cdot \mathrm{km}^{-1}\end{array}$ & $\begin{array}{c}-185420 \\
\mathrm{ps} \cdot \mathrm{nm}^{-1} \cdot \mathrm{km}^{-1}\end{array}$ \\
\hline Phase-matching $\lambda$ & $1.5964 \mu \mathrm{m}$ & $1.5999 \mu \mathrm{m}$ \\
\hline Hole's diameter $\mathrm{d}$ & $\begin{array}{c}\text { Optimal: } \\
11.7051 \mu \mathrm{m}\end{array}$ & $\begin{array}{c}11.7051 \mu \mathrm{m} \\
(\text { constant })\end{array}$ \\
\hline Hole's pitch $\Lambda$ & $\begin{array}{c}23.2 \mu \mathrm{m} \\
(\text { constant })\end{array}$ & $\begin{array}{c}23.2 \mu \mathrm{m} \\
(\text { constant) }\end{array}$ \\
\hline $\begin{array}{c}\text { Normalized hole } \\
\text { diam. } \mathrm{d} / \Lambda\end{array}$ & $\begin{array}{c}\text { Optimal: } \\
0.504530\end{array}$ & $\begin{array}{c}0.50453 \\
(\text { constant })\end{array}$ \\
\hline Bending radius $\mathrm{R}$ & $\begin{array}{c}66116 \mu \mathrm{m} \\
(\text { constant) }\end{array}$ & $\begin{array}{c}\text { Optimal: } \\
69080 \mu \mathrm{m}\end{array}$ \\
\hline
\end{tabular}

\section{SIMULATION RESULTS AND DISCUSSION}

The following section presents the results of simulations of bending effects in selected types of microstructured fibers, primarily focused on the mutual relations of minimum dispersion, minimum- dispersion wavelength, bending radius, and normalized hole diameter. The sensitivity of modeled quantities to deviations of fiber geometry is discussed.

\subsection{Coupling modes in bent microstructured fiber}

Compared to the properties of straight microstructured fiber, the fundamental mode field distribution is not radial, since bending strongly affects the shape of the fundamental mode, which is depicted in Fig. 2 (left). For bending stronger than the specific critical bending, the optical power is coupled into the cladding. Bending is responsible for the presence of coupling modes in the spaces between adjacent holes, which is in agreement with Fig. 2 (right). The core-mode exhibits negative dispersion over a short wavelength interval, (Fig. 3). For a clearer view, chromatic dispersion as a function of wavelength for the straight fiber is plotted in Fig. 4. The resultant dispersion for the core-guided mode is $-185000 \mathrm{ps} \cdot \mathrm{nm}^{-}$ ${ }^{1} \cdot \mathrm{km}^{-1}$ at the wavelength of $1559.9 \mathrm{~nm}$. This is possible only for the suitable configuration of the value of bending radius, combined with the specific set of structural parameters, as displayed in Table 1 . It must be said that since at least the value of one of the parameters differs from the predicted value, there is no negative chromatic dispersion.
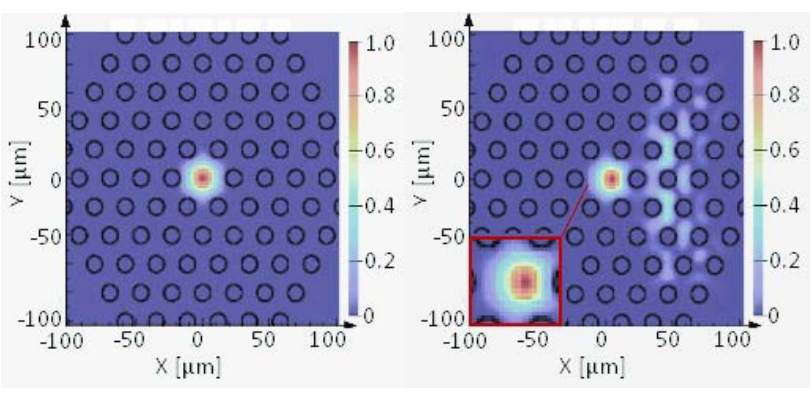

Fig. 2 Comparison of the mode distribution for the straight PCF (left) and for the PCF bent at the radius of $69.1 \mathrm{~mm}$ (right)

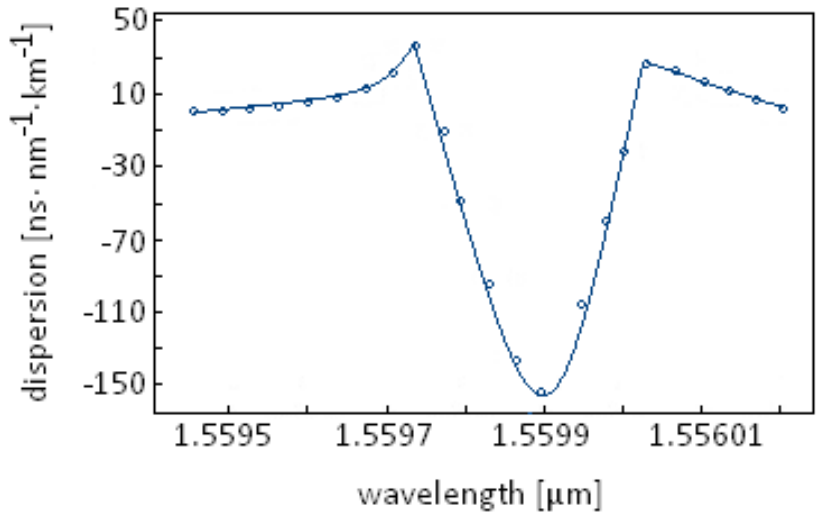

Fig. 3 Chromatic dispersion diagram of modeled PCF bent at the radius of $66.1 \mathrm{~mm}$

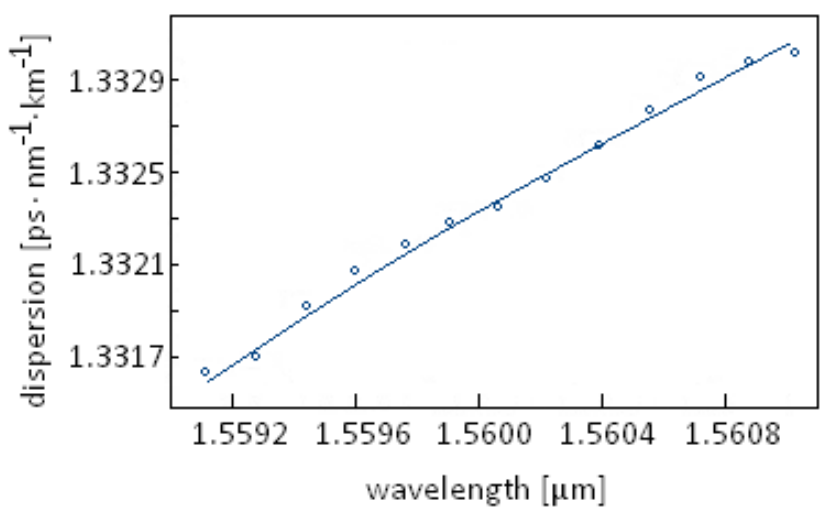

Fig. 4 Chromatic dispersion diagram calculated for unbent PCF

Coupling between modes is possible at the specific phase-matching wavelength, and is accompanied by the nonlinear evolution of effective index, according to Fig. 5 and Fig. 6. The observation of mode distribution and effective index is a feedback for predicting the desired dispersion properties, being as well a suitable tool to reduce the simulation time, since the perspective to achieve negative values of chromatic dispersion is estimated. On the contrary to the unbent structure, the effective index upon wavelength in bent PCF is nonlinear. The phase matching wavelength is at the C-band, which can be observed from Fig. 5 and Fig. 6 .

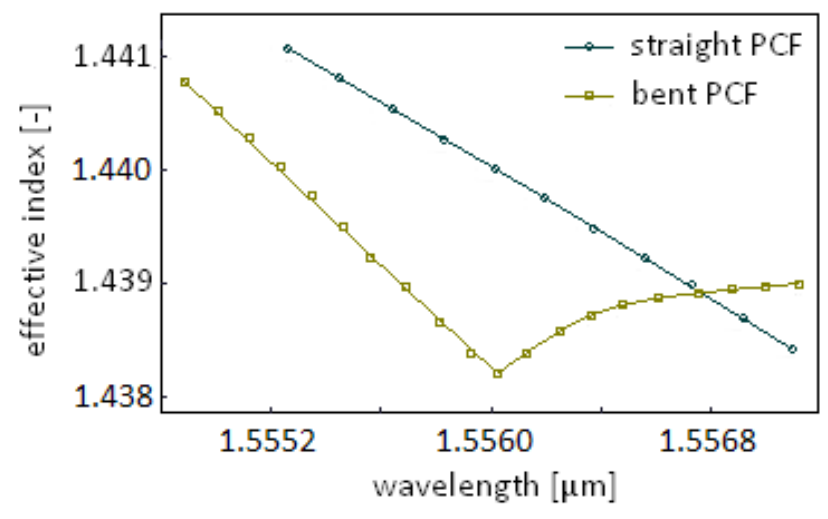

Fig. 5 Effective index of the fundamental mode in straight PCF and in the PCF bent at the radius of $69.1 \mathrm{~mm}$ 


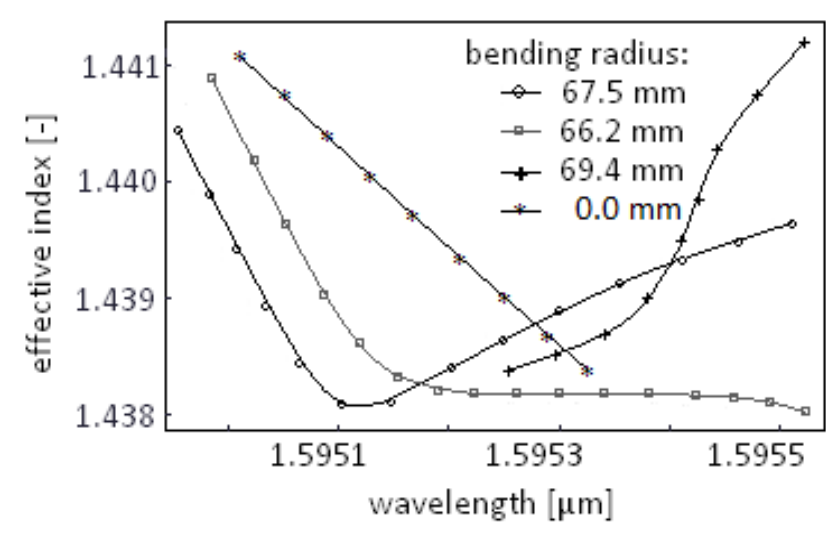

Fig. 6 Sample evolution of effective index indicating the existence of coupling from the fundamental core-mode to the gallery of cladding modes obtained for different bending

\subsection{Negative chromatic dispersion resulting from fiber bending}

In classical dual-core fibers, there is significant positive dispersion, related to the cladding mode [16]. In PCFs, it is suppressed, because instead of one cladding mode, there is a gallery of low-intensity cladding modes. To maintain huge negative chromatic dispersion for some other value of bending radius, the value of normalized hole diameter should be adjusted.

In addition, strong dependence of coupling on the presence of specific holes indicates that the investigation of the influence of fiber's geometry deviations on the resultant minimum dispersion is necessary. Fig. 7 shows the wavelength evolution of chromatic dispersion, (primarily of the minimum dispersion) as a function of bending radius for fixed value of normalized hole diameter $(d / \Lambda=0.50453)$. The greater radius of curvature (smaller bending) is responsible for the occurrence of minimum dispersion at shorter wavelength. The largest minimum dispersion is obtained at $1560 \mathrm{~nm}$ for the normalized hole diameter being 0.50453 and obtained for the PCF bent at the radius of $69.1 \mathrm{~mm}$. The theoretical chromatic dispersion is $-185000 \mathrm{ps} \cdot \mathrm{nm}^{-1} \cdot \mathrm{km}^{-1}$. The bandwidth, for which chromatic dispersion is negative, is not flat and is very narrow $(0.07 \mathrm{~nm})$, resulting as well in huge dispersion slope. The minimum dispersion at $1550 \mathrm{~nm}$ is $-87000 \mathrm{ps} \cdot \mathrm{nm}^{-1} \cdot \mathrm{km}^{-1}$ and is obtained for the curvature radius of $69.9 \mathrm{~mm}$, the normalized hole diameter is 0.50453 . The effective mode area calculated for the straight fiber is $314.7 \mu \mathrm{m}^{2}$. The effective mode area in the bent PCF, calculated for the core-mode is 700 $\mu \mathrm{m}^{2}$, which is relevant, because light coupled from the core or reshaped by reason of bending is not taken into account for the calculation; with coupled light, the mode area is $2690 \mu \mathrm{m}^{2}$. Since the value of bending radius is varied by $1 \%$ towards smaller values than the reference one (smaller value of bending radius denotes stronger bending), the peak value of chromatic dispersion occurs at the 7-nm-longer wavelength, and it is $35 \%$ smaller. If bending radius has equivalent positive deviation, the minimum dispersion occurs at wavelength shorter than the reference one, and the value of the minimum dispersion is more than half smaller ( $57 \%$ decrease). The peak value of dispersion is found to be less immune to the positive deviation in bending radius (positive deviation denotes bending radius greater than the predicted one) compared to identical negative deviation. The value of normalized hole diameter and predicted value of bending radius plays an important role for the occurrence of negative chromatic dispersion. The minimum dispersion wavelength varies linearly upon bending radius, as depicted in Fig. 8. Similar behavior refers to the zero-dispersion wavelength, which is longer than the minimum dispersion wavelength.

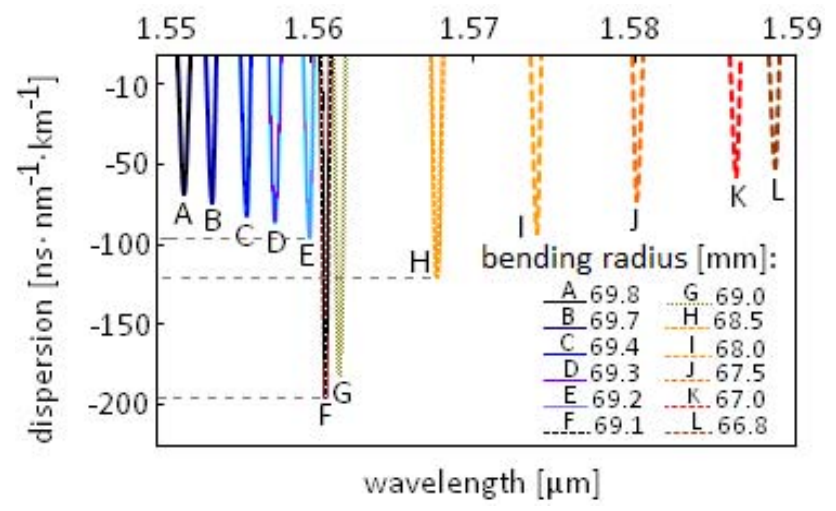

Fig. 7 Minimum values of chromatic dispersion at different wavelengths for varied bending radius and fixed value of hole diameter $\mathrm{d} / \Lambda=0.50453$

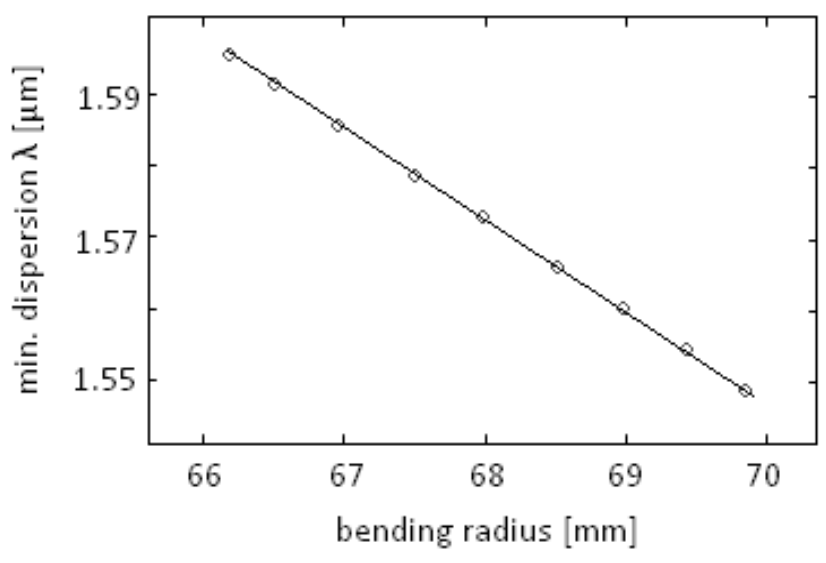

Fig. 8 Evolution of minimum-dispersion wavelength upon bending radius

Fig. 9 shows that the minimum dispersion is suppressed and occurs at shorter wavelength for the hole diameter being greater than the predicted value, whereas the minimum dispersion wavelength is tuned to longer wavelengths and the peak value of chromatic dispersion demonstrates a remarkable variation since the hole diameter is smaller. For the negative deviation in hole diameter, the peak value of chromatic dispersion decreases by $74 \%$ (with the shift of $1.5 \mathrm{~nm}$ towards longer wavelengths). On the contrary, it decreases by $90 \%$ for equivalent positive deviation. It can be concluded that negative tolerance to the hole diameter is more acceptable (holes manufactured being smaller than the designed ones) than the positive one. To precisely tune the minimum dispersion wavelength, for example in the design of DCF, the greater tolerance to the normalized hole diameter than to the bending radius can be assumed. To tune the minimum dispersion to other wavelength, the bending radius has to be adjusted, and the value of normalized hole diameter must be predicted again. Bending radius is found 
to be an important factor for tuning the minimum negative dispersion wavelength, whereas the value of normalized hole diameter is responsible primarily for the peak value of chromatic dispersion.

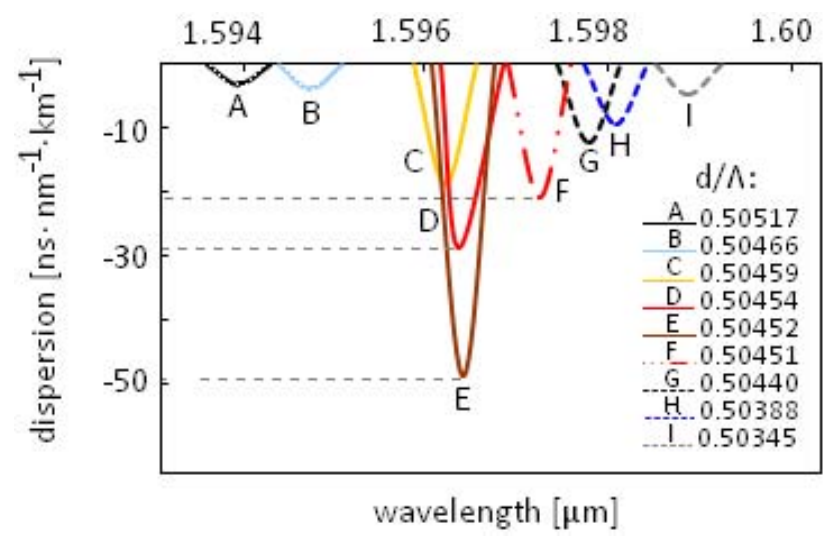

Fig. 9 Minimum values of dispersion as a function of normalized hole diameter for fixed value of bending radius $(66.1 \mathrm{~mm})$

Nevertheless, the stability of the value of chromatic dispersion exactly at the $1550-\mathrm{nm}$ wavelength is even more essential problem. It is determined by the following features. The first feature is the peak value being smaller for a given deviation of fiber's geometry, (identical deviation of each hole is assumed). Furthermore, the dispersion value at $1550 \mathrm{~nm}$ is not the peak value (the peak value occurs at different wavelength). The suppression by $20000 \mathrm{ps} \cdot \mathrm{nm}^{-1} \cdot \mathrm{km}^{-1}$ at the $1550 \mathrm{~nm}$ corresponds to the $5 \mu \mathrm{m}$ deviation in bending radius, which is displayed in Fig. 10. Let's assume the hole diameter being $d / \Lambda=0.50453$ and the value of bending radius of $69.9 \mathrm{~mm}$. By introducing the negative deviation to one hole $(d / \Lambda=0.4914)$ close to the cladding field, the chromatic dispersion at $1.55 \mu \mathrm{m}$ is suppressed by $30 \%$. For the comparison, the same deviation for all holes results in the peak value being ten times smaller. Otherwise, the imprecision of holes in regions far from coupling modes have marginal importance for the value of chromatic dispersion.

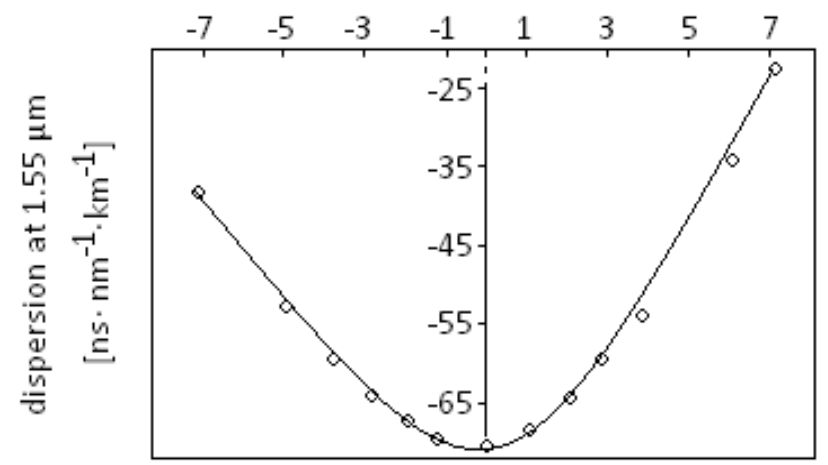

bending radius deviation $[\mu \mathrm{m}]$

Fig. 10 Chromatic dispersion at $1550 \mathrm{~nm}$ for different deviation in bending radius

The conclusions about potential tuning of minimum dispersion wavelength (as well as zero-dispersion wavelength) indicate that the bending mechanism can tune an operating wavelength, if only the radius of reel is suitable. There is necessity to take into account the possible shift of operating wavelength after winding the fiber onto a reel. Another approach is that the operating wavelength can be accurately tuned by right selection of the reel radius. The smaller the radius of winding is, the greater the tune of an operating wavelength is.

\section{CONCLUSIONS}

The contribution of this paper is the detailed, complex study of bending results, determined in a solid-core PCF. Negative chromatic dispersion is produced only for the suitable configurations of the structural parameters combined with specific bending. As far as practical consideration is concerned, the presented work explains the consequences of fiber bending that can affect optical fibers in standard office environment. There is, for example, the potential change in transmission diagrams of PCFs at the C-band. Smaller curvature radius (stronger bending) is responsible for tuning the minimum dispersion wavelength towards longer wavelengths. Decreased normalized hole diameter tunes minimum dispersion into longer wavelengths.

The future work on the problem deals with the design of a PCF immune to bending, using the presented knowledge. Another approach could employ fiber bending to potential switching of the modes between the fiber's core-mode and the possible cladding defect mode. PCFs with ring structure in the cladding could be investigated, too. For this purpose, the confinement of cladding modes in the bent PCF into one cladding mode should be done.

\section{ACKNOWLEDGMENT}

This work is supported by the Grant Agency of the Czech Republic under project 102/09/P143.

\section{REFERENCES}

[1] FEVRIER, S. - AUGuSte, J. - BLONDY, J. PEYRILLOUX, A. - ROY, P. - PAGNOUX, D.: Accurate Tuning of the Highly-Negative Chromatic Dispersion Wavelength Into a dual Concentric Core Fibre by Macro-Bending, ECOC'02, Fibers and Waveguide Components, P1. 08, 2002.

[2] WANG, Q. - FARRELL, G. - FREIR, T.: Theoretical and Experimental Investigations of Macro-Bend Losses for Standard Single Mode Fibers, Opt. Express 13, 4476-4484, 2005.

[3] FAUSTINI, L. - MARTINI, G.: Bend loss in singlemode fibers, J. Lightwave Technol. 15, 671-679, 1997.

[4] NIELSEN, M. - MORTENSEN, N. ALBERTSEN, M. - FOLKENBERG, J. BJARKLEV, A. - BONACINNI, D.: Predicting Macrobending Loss for Large-Mode Area Photonic Crystal Fibers, Opt. Express 19, 1775-1779, 2004.

[5] BAGGETT, J. - MONRO, T. - FURUSAWA, K. FINAZZI, V. - RICHARDSON, D.: Understanding 
Bending Losses in Holey Optical Fibers, Opt. Commun. 227, pp. 317-335, 2005.

[6] TSUCHIDA, Y. - SAITOH, K. - KOSHIBA, M.: Design and Characterization of Single-Mode Holey Fibers with Low Bending Losses, Opt. Exp. 4770, Vol. 13, No.12, 2005.

[7] OLSZEWSKI, J. - SZPULAK, M. MARTYNKIEN, T. - URBANCZYK, W.: Effect of Coupling between Fundamental and Cladding Modes on Bending Losses in Photonic Crystal Fibers, Opt. Express 6015, Vol. 13, No. 16, 2005.

[8] EIJKELENBORG, M. - CANNING, J. - RYAN, T. - LYYTIKAINEN, K.: Bending-Induced Colouring in a Photonic Crystal Fibre, Opt. Express 88, Vol. 7, No. 2, 2000.

[9] GU, X. - BLANK, L.: $10 \mathrm{Gbit} / \mathrm{s}$ Unrepeatered Three-level Optical Transmission over $100 \mathrm{~km}$ of Standard Fibre, Electronics Letters, Vol. 29, 1993.

[10] ITU-T G.652, recommendation for: transmission systems and media, digital systems and networks, Optical fibre cables, characteristics of a single-mode optical fibre and cable.

[11] ZHANG, Y. - YANG, S. - PENG, X. - LU, Y. CHEN, X. - XIE, S.: Design of Large Effective Area Microstructured Optical Fiber for Dispersion Compensation, Photonic Crystals and Fibers. Bellingham: SPIE, Vol. 5950, No. 43, 2005.

[12] HUTTUNEN, A.: Optimization of Dual-core and Microstructure Fiber Geometries for Dispersion Compensation and Large Mode Area, Opt. Express 627, Vol. 13, No. 2, 2005.

[13] FUJISAWA, T. - SAITOH, K. - WADA, K. KOSHIBA, M.: Chromatic Dispersion Profile Optimization of Dual-concentric-core Photonic Crystal Fibers for Broadband Dispersion Compensation, Opt. Express 893, Vol. 14, No. 2, 2006.

[14] YANG, S. - ZHANG, Y. - PENG, X. - LU, Y. XIE, S. - LI, J. - CHEN, W. - JIANG, Z. - PENG, J. - LI, H.: Theoretical Study and Experimental Production of High Negative Dispersion Photonic Crystal Fiber with Large Area Mode Field, Opt. Express 3015, Vol. 14, No. 7, 2006.

[15] ZSIGRI, B. - LÆGSGAARD, J. - BJARKLEV, A.: A Novel Photonic Crystal Fibre Design for Dispersion Compensation, J. Opt. A: Pure Appl. Opt. 6, pp. 717-720, 2004.

[16] GEROME, F. - AUGUSTE, J. - MAURY, J. BLONDY, J. - MARCOU, J.: Theoretical and Experimental Analysis of a Chromatic Dispersion Compensating Module Using a Dual Concentric Core Fiber, J. Lightwave Technol., Vol. 24, No. 1, 2006.

[17] LUCKI, M. - BOHAC, L. - BURCIK, J.: Chosen Aspects of Negative Dispersion Generation in Index Guiding Photonic Crystal Fibers, SPIE, Photonic Crystal Fibers, Vol. 6588, 2007.
[18] LUCKI, M.: Negative Chromatic Dispersion in Selected Types of Photonic Crystal Fibres 24. Obtained by Bending, Proceedings of the 11th International Conference on Transparent Optical Networks, p.Tu.B1.6. ISBN 978-1-4244-4826-5, 2009.

[19] COZENS, J. - BOUCOUVALAS, A.: Coaxial Optical Coupler, Electron. Lett., Vol. 18, No. 3, pp. 138-140, 1982.

[20] BOUCOUVALAS, A.: Coaxial Optical Fiber Coupling, J. Lightwave Technol., Vol. LT-3, No. 5, pp. 1151-1158, 1985.

[21] AUGuSte, J. - BLONDY, M. - MAURY, J. MARCOU, J. - DUSSARDIER, B. - MONNOM, G. - JINDAL, R. - THYAGARAJAN, K. - PAL, B.: Conception, Realization, and Characterization of a Very High Negative Chromatic Dispersion Fiber, Opt. Fiber Technol., Vol. 8, No. 1, pp. 89-105, 2002.

[22] QIU, M.: Analysis of Guided Modes in Photonic Crystal Fibers Using the Finite-Difference TimeDomain Method, Microwave Opt. Technol. Lett. 30, pp. 327-330, 2001.

[23] YU, C. - CHANG, H.: Compact Finite-Difference Frequency-Domain Method for the Analysis of TwoDimensional Photonic Crystals, Opt. Express 1397, Vol. 12, No. 7, 2004.

[24] KAWANO, K. - KITOH, T.: Introduction to Optical Waveguide Analysis, Solving Maxwell's Equations and the Schroedinger Equation, A. Wiley Interscience Publication, John Wiley \& Sons, Inc., New York, 2001.

[25] ZHU, Z. - BROWN, T.: Full-Vectorial FiniteDifference Analysis of Microstructured Optical Fibers, Opt. Express 853, Vol. 10, No. 17, 2002.

[26] YEE, K.: Numerical Solution of Initial Boundary Value Problems Involving Maxwell's Equations in Isotropic Media, IEEE Trans. Antennas Propagat. 14, pp. 302-307, 1966.

[27] ZOLLA, F. - RENVERSEZ, G. - NICOLET, A. KUHLMEY, B.: Foundations of Photonic Crystal Fibers, Imperial College Press, ISBN 1-86094-5074, 2005 .

Received December 15, 2010, accepted April 20, 2011

\section{BIOGRAPHY}

Michal Lucki is now a researcher and a lecturer at the Department of Telecommunications Engineering, Faculty of Electrical Engineering of the Czech Technical University in Prague. He received his $\mathrm{PhD}$ degree in 2007 at this university. His current research activities include optical communications, photonics, Photonic Crystal Fibers, which are the subject of investigation within the framework of number of projects, which are sponsored by the Grant Agency of the Czech Republic. 